NISSUNA UMANA INVESTIGAZIONE SI PUO DIMANDARE VERA SCIENZIA S'ESSA NON PASSA PER LE MATEMATICHE DIMOSTRAZIONI LEONARDO DA VINCI

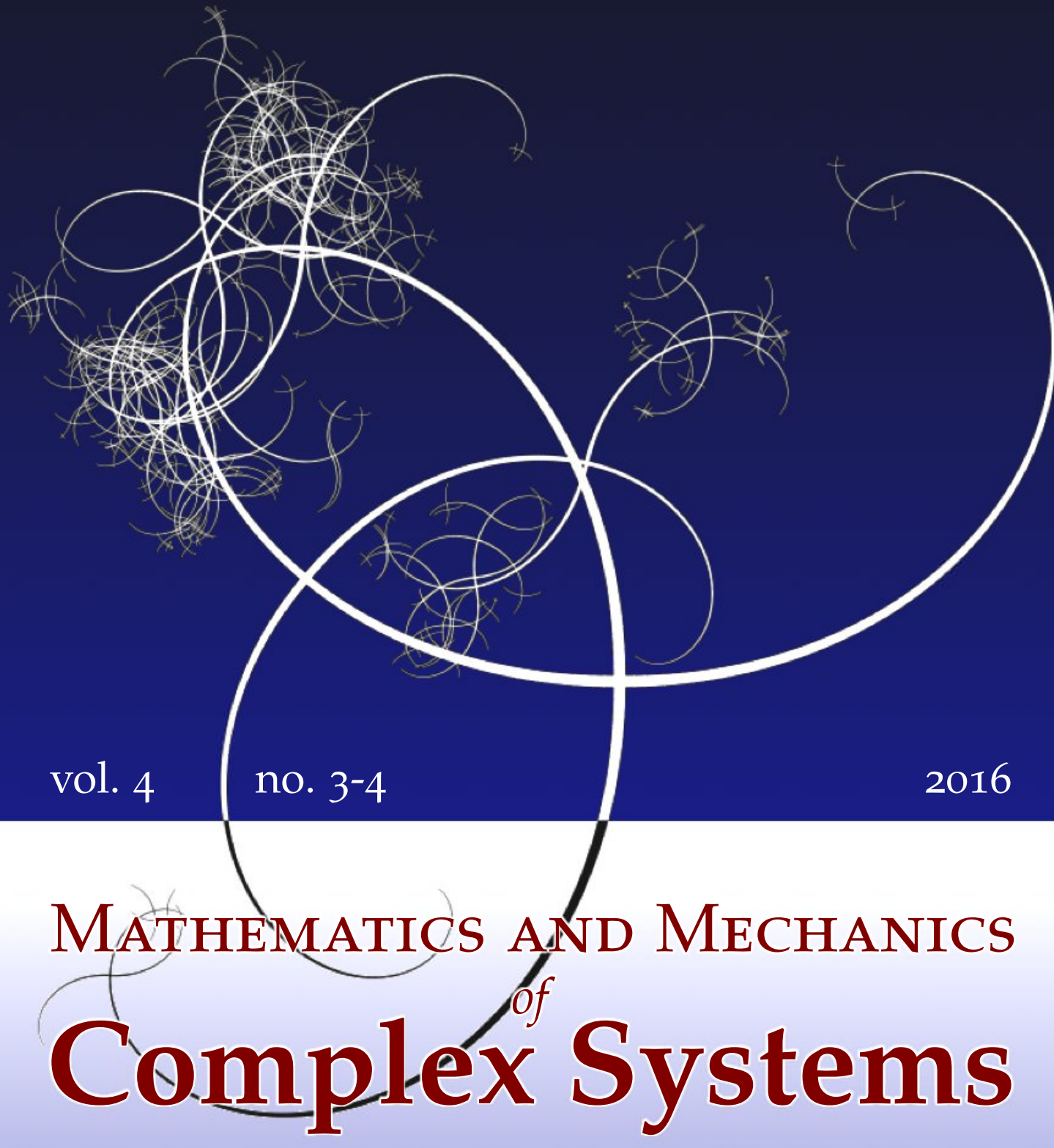

FIORELLA BARONE AND SANDRO GRAFFI A REMARK ON EIGENVALUE PERTURBATION THEORY AT VANISHING ISOLATION DISTANCE 


\title{
A REMARK ON EIGENVALUE PERTURBATION THEORY AT VANISHING ISOLATION DISTANCE
}

\author{
FIORELLA BARONE AND SANDRO GRAFFI
}

\begin{abstract}
Let $T$ be a self-adjoint operator in a separable Hilbert space $X$, admitting compact resolvent and simple eigenvalues with possibly vanishing isolation distance, and let $V$ be symmetric and bounded. Consider the self-adjoint operator family $T(g): g \in \mathbb{R}$ in $X$ defined by $T+g V$ on $D(T)$. A simple criterion is formulated ensuring, for any eigenvalue of $T(g)$, the existence to all orders of its perturbation expansion and its asymptotic nature near $g=0$, with estimates independent of the eigenvalue index. An application to a class of Schrödinger operators is described.
\end{abstract}

\section{Introduction and formulation of the result}

The standard Rellich-Kato regular perturbation theory [Kato 1976] applies to isolated eigenvalues of finite multiplicity of a densely defined, closed operator $T$ in a Banach space $X$. We consider here only the particular case in which

- $X$ is a separable Hilbert space,

- $T$ is a self-adjoint operator in $X$ with compact resolvent and simple spectrum, and

- the perturbation is symmetric, regular and linear on the perturbation parameter.

Let the operator $V: D(V) \rightarrow X$ be symmetric and $T$-bounded with relative bound $b$; i.e., let $D(T) \subset D(V)$, and let there exist $a>0$ and $b>0$ such that

$$
\|V u\| \leq b\|T u\|+a\|u\| \quad \text { for all } u \in D(T) .
$$

With $g \in \mathbb{C}$, consider the operator family in $X$ defined as

$$
g \mapsto T(g):=T u+g V u, \quad D(T(g))=D(T) .
$$

Then $T(g)$ is closed with nonempty resolvent set for $|g|<1 / b$ and $T(g)^{*}=T(\bar{g})$ so that $T(g)=T(g)^{*}$ if $g \in \mathbb{R}$. Let $\lambda \in \mathbb{R}$ be an isolated eigenvalue of $T$ (simple by the above assumption), with isolation distance $d(\lambda)>0$. Here, let us recall that

$$
d(\lambda):=\operatorname{dist}(\operatorname{Spec}(T) \backslash\{\lambda\}, \lambda)>0 .
$$

Communicated by Raffaele Esposito.

MSC2010: 81Q05, 81Q10, 81Q15.

Keywords: isolation distance, eigenvalue perturbation theory. 
Then for $|g|$ suitably small, $T(g)$ has one and only one simple eigenvalue $\lambda(g)$ such that $\lim _{g \rightarrow 0} \lambda(g)=\lambda$ (see, e.g., [Kato 1976, §§VII.2-3] or [Reed and Simon 1978, §XII.1]). The function $g \mapsto \lambda(g)$ is holomorphic in a disk centered at the origin because its Taylor expansion at $g=0$

$$
\lambda(g)=\lambda+\sum_{\ell=1}^{\infty} \lambda_{\ell} g^{\ell}
$$

exists and converges for $|g|<r_{d}(\lambda)$, with $r_{d}(\lambda)>0$. The coefficients $\lambda_{\ell}$ are generated by (Rayleigh-Schrödinger) perturbation theory. This existence and convergence result depends in a critical way on the positivity of $d(\lambda)$ and therefore does not apply to nonisolated eigenvalues.

To the best of our knowledge, a simple, explicit criterion ensuring existence, let alone convergence, of (Rayleigh-Schrödinger) perturbation theory when $r_{d}(\lambda) \rightarrow 0$ is still missing, even under much stronger assumptions such as $r_{d}(\lambda) \rightarrow 0$ only if $\lambda \rightarrow \infty$ and boundedness of $V$. (For related questions involving the behavior of $r_{d}(\lambda)$ as $\lambda \rightarrow \infty$, we refer the reader to [Reed and Simon 1978, §XIII.5] and to [Brownell and Clark 1961; McLeod 1961; Tamura 1974]). Within this last class of Hilbert space operators, we formulate and prove here a similar criterion, working out the necessary estimates on the behavior of $\lambda_{\ell}$ uniform with respect to the eigenvalue index $\underline{n}$. Under more restrictive assumptions on the vanishing of $r_{d}(\lambda)$, the explicit dependence on $\underline{n}$ of the above estimates is actually determined.

Our hypotheses are formulated as follows.

(A1) $T$ is a nonnegative self-adjoint operator in the separable Hilbert space $X$, with compact resolvent and simple spectrum. Its eigenvalues are denoted by $\left\{\lambda_{\underline{n}}: \underline{n} \in \mathbb{N}^{s}\right\}, s \geq 1$, and the corresponding (normalized) eigenvectors by $\left\{\psi_{\underline{n}}: \underline{n} \in \mathbb{N}^{s}\right\}$.

(A2) $d(\underline{m}, \underline{n}):=\left|\lambda_{\underline{m}}-\lambda_{\underline{n}}\right| \rightarrow 0$ if and only if $|\underline{m}-\underline{n}| \rightarrow \infty$. Here $|n|:=n_{1}+\cdots+n_{s}$, $\underline{n} \in \mathbb{N}^{s}$.

(A3) There are $\Lambda>0$ and $\gamma>s-1$ such that

$$
\left|\lambda_{\underline{m}}-\lambda_{\underline{n}}\right|^{-1} \leq \Lambda|\underline{m}-\underline{n}|^{\gamma}, \quad \underline{m} \neq \underline{n} .
$$

Here $|\underline{x}|^{\gamma}:=x_{1}^{\gamma}+\cdots+x_{s}^{\gamma}$.

(A4) $V: X \rightarrow X$ is symmetric and bounded (hence self-adjoint). Moreover, there exist $A>\alpha$ and $\alpha>0$ such that

$$
\left|\left\langle\psi_{\underline{m}}, V \psi_{\underline{n}}\right\rangle\right| \leq A e^{-\alpha|\underline{m}-\underline{n}|}, \quad|\underline{m}-\underline{n}| \rightarrow \infty .
$$

Since $V$ is bounded, it is a fortiori $T$-bounded with $T$-bound 0 . Thus, the operator family $g \mapsto T(g)=T+g V$ with $D(T(g))=D(T)$ is type-A real-holomorphic in the sense of Kato [1976, §VII.2] for all $g \in \mathbb{C}$. 
Consider now the Rayleigh-Schrödinger perturbation expansion near any eigenvalue $\lambda_{\underline{n}}(g)$ of $H(g)$. The initial point of $\lambda_{\underline{n}}(g)$ is the eigenvalue $\lambda_{\underline{n}}$ of $T, \underline{n} \in \mathbb{Z}^{s}$. Thus,

$$
\lambda_{\underline{n}}(g)=\lambda_{\underline{n}}+\sum_{\ell=1}^{\infty} B_{\ell}(\underline{n}) g^{\ell} .
$$

The expansion (1-7) has positive radius of convergence $r_{n}, \underline{n} \in \mathbb{Z}^{s}$, by the boundedness of $V$, which implies

$$
r_{\underline{n}} \geq \frac{d\left(\lambda_{\underline{\underline{n}}}\right)\|V\|}{2}, \quad d\left(\lambda_{\underline{n}}\right)=\operatorname{dist}\left(\operatorname{Spec}\left[(T) \backslash\left\{\lambda_{\underline{n}}\right\}\right], \lambda_{\underline{n}}\right) .
$$

The vanishing of the convergence radius $r(\underline{n}) \downarrow 0$ as $d\left(\lambda_{\underline{n}}\right) \downarrow 0$ not only may cause the divergence of the perturbation expansion but may prevent its very existence also for a bounded perturbation $V$; see Remarks 1.3 and 2.1. Then the purpose of this paper is to explicit determine, under the above assumptions, the dependence of the perturbation series on the vanishing rate of the isolation distance $d\left(\lambda_{n}\right)$ by proving the following quantitative estimate.

Theorem 1.1. Let $T$ and $V$ fulfill assumptions (A1)-(A3). Set

$$
R(\Lambda, \alpha, \gamma):=\frac{\Lambda}{\alpha^{\gamma}} .
$$

Then the following $\underline{n}$-independent estimate holds:

$$
\left|B_{\ell}(\underline{n})\right|<R(\Lambda, \alpha, \gamma)^{\ell}(4 \ell)^{\ell+1}[\gamma(\ell-1)] \text { ! for all } \underline{n} \in \mathbb{Z}^{s} .
$$

The uniform estimate (1-10) makes it possible to establish the uniform asymptotic nature to all orders of the perturbation expansion.

Corollary 1.2. The perturbation expansion $\lambda_{\underline{n}}+\sum_{\ell=1}^{\infty} B_{\ell}(\underline{n}) g^{\ell}$ represents an asymptotic expansion to all orders of the eigenvalue $\lambda_{\underline{n}}(g)$ uniformly with respect to $\underline{n} \in \mathbb{N}^{s} ;$ i.e, for any fixed $N \in \mathbb{N}$,

$$
\lim _{|g| \rightarrow 0} \frac{\left|\lambda_{\underline{n}}(g)-\sum_{\ell=1}^{N} B_{\ell}(\underline{n}) g^{\ell}\right|}{|g|^{N}}=0
$$

uniformly with respect to $\underline{n} \in \mathbb{N}^{s}$.

Remark 1.3. The very existence of perturbation theory at the vanishing of the isolation distance, i.e., at the limit $\underline{n} \rightarrow \infty$ in the present case, requires the validity of estimates independent of $\underline{n}$ on the coefficients $B_{\ell, \underline{n}}$. The conditions (1-5) and (1-6) imply the existence of $\bar{g}(\underline{n})>0$ such that

$$
|g| \sum_{\substack{\underline{m} \in \mathbb{Z}^{s} \\ \underline{m} \neq \underline{n}}} \frac{\left|\left\langle\psi_{\underline{m}}, V \psi_{\underline{n}}\right\rangle\right|}{\left|\lambda_{\underline{m}}-\lambda_{\underline{n}}\right|}<1, \quad|g|<\bar{g}(\underline{n}) .
$$


Now

$$
\sup _{\underline{m} \neq \underline{n} \underline{\underline{n}}} \frac{1}{\left|\lambda_{\underline{m}}-\lambda_{\underline{n}}\right|} \leq \frac{2}{d_{\underline{n}}} .
$$

Moreover, since $V$ is bounded and symmetric,

$$
\|V\|=\max \left(\sup _{\underline{m} \in \mathbb{Z}^{s}} \sum_{n \in \mathbb{Z}^{s}}\left|\left\langle\psi_{\underline{m}}, V \psi_{\underline{n}}\right\rangle\right|, \sup _{\underline{n} \in \mathbb{Z}^{s}} \sum_{\underline{m} \in \mathbb{Z}^{s}}\left|\left\langle\psi_{\underline{m}}, V \psi_{\underline{n}}\right\rangle\right|\right),
$$

whence

$$
\sum_{\substack{m \in \mathbb{Z}^{s} \\ \underline{m} \neq \underline{\underline{n}}}} \frac{\left|\left\langle\psi_{\underline{m}}, V \psi_{\underline{n}}\right\rangle\right|}{\left|\lambda_{\underline{m}}-\lambda_{\underline{n}}\right|} \leq \frac{2}{d_{\underline{n}}}\|V\| .
$$

Thus, if $\underline{n}$ is fixed, i.e., if $\lambda_{\underline{n}}$ is isolated with isolation distance $d_{\underline{n}}>0$, the standard convergence criterion valid for the perturbation series of the isolated eigenvalue $\lambda_{\underline{n}}$ under the bounded perturbation $V$ (see, e.g., [Kato 1976, §VII.2])

$$
\frac{2|g|}{d_{\underline{n}}}\|V\|<1
$$

implies a fortiori the inequality (1-12). On the other hand, as $\underline{n} \rightarrow \infty$, i.e., $d_{\underline{n}} \rightarrow 0$, in general the inequality (1-13) has a meaning only for $g=0$, while (1-12) can be rewritten (again by (1-5) and (1-6), which are an adaptation of the small-denominator conditions of classical perturbation theory) in the form of an inequality independent of $\underline{n}$ :

$$
|g| A \Lambda\left[\frac{d^{\gamma}}{d \alpha^{\gamma}}\left(2 e^{\alpha \gamma}\right)\right]^{s}<1 .
$$

This inequality is the starting point for the $\underline{n}$-independent estimates of Theorem 1.1. Remark 1.4 (notation). The underlining operation always transforms into vector indices with $s$ components the corresponding scalar ones. Namely,

$$
\underline{m}:=\left(m_{1}, \ldots, m_{s}\right) \in \mathbb{Z}^{s}, \quad m_{i} \in \mathbb{Z}, i=1, \ldots, s .
$$

The star operation transforms a positive integer index into a nonnegative one; i.e., $q \in \mathbb{N}_{*}$ means $q=0,1, \ldots$. Furthermore, $|\underline{m}|$ denotes the length of the multiindex $\underline{m}$ :

$$
|\underline{m}|:=\left|m_{1}\right|+\cdots+\left|m_{s}\right| .
$$

Products and powers of multi-indices abbreviate products and powers of the composing indices:

$$
\begin{aligned}
\underline{m} ! & :=m_{1} ! \cdots m_{s} !, \\
\underline{z}^{\underline{m}} & :=z_{1}^{m_{1}} \cdots z_{s}^{m_{s}}, \\
\Gamma(\alpha \underline{z}) & :=\Gamma\left(\alpha z_{1}\right) \cdots \Gamma\left(\alpha z_{s}\right), \quad \alpha \in \mathbb{R} .
\end{aligned}
$$


Example 1.5. Let $T$ be the Schrödinger operator in $L^{2}\left(\mathbb{R}^{s}\right)$ with domain and action defined as

$$
\begin{aligned}
D(T) & =H^{2}\left(\mathbb{R}^{s}\right) \cap L_{2}^{2}\left(\mathbb{R}^{s}\right), \\
T u & =-\frac{1}{2} \Delta u+\frac{1}{2} \sum_{k=1}^{s}\left[\omega_{k}^{2} x_{k}^{2}-s / 2\right] u, \quad u \in D(T) .
\end{aligned}
$$

$T$ is the self-adjoint, compact-resolvent Schrödinger operator generated by the $p$ dimensional quantum harmonic oscillator, with frequencies $1 \geq \omega_{k}>0, k=1, \ldots, s$. Thus, condition (A1) is fulfilled.

The rescaling map $\left(U_{\underline{\omega}} f\right)(\underline{x})=\left(\omega_{1} \cdots \omega_{s}\right)^{1 / 2} f(\underline{\omega x}), \underline{\omega x}:=\left(\omega_{1} x_{1}, \ldots, \omega_{s} x_{s}\right)$, is unitary in $L^{2}\left(\mathbb{R}^{s}\right)$, and by an abuse of notation, we still denote by $T$ the unitary image $U_{\underline{\omega}} T U_{\omega}^{-1}$. Hence, the action $T u$ becomes

$$
T u=\frac{1}{2} \sum_{k=1}^{s} \omega_{k}\left[-\frac{d^{2} u}{d x_{k}^{2}}+x_{k}^{2} u-I u\right] .
$$

The corresponding eigenvalues are

$$
\lambda_{\underline{n}}(\omega)=\sum_{k=1}^{s} \omega_{k} n_{k}:=\langle\underline{\omega}, \underline{n}\rangle, \quad \underline{n} \in\left(\mathbb{N}_{*}\right)^{s} .
$$

Since $\omega_{i}>0$ and $n_{i}>0, i=1, \ldots, s$, the difference $\left|\lambda_{\underline{r}}-\lambda_{\underline{n}}\right|=\langle\underline{\omega},(\underline{r}-\underline{n})\rangle$ can vanish only if $|\underline{r}-\underline{n}| \rightarrow \infty$, with at least two of the components $r_{j}-n_{j}$, $j=1, \ldots, s$, having different sign. Hence, condition (A2) is fulfilled. Assume now irrational independence of the frequencies, i.e.,

$$
\omega_{1} v_{1}+\cdots+\omega_{s} v_{s}=0, v_{k} \in \mathbb{Z}, \quad \text { if and only if } v_{k}=0, k=1, \ldots, s .
$$

Then all eigenvalues $\lambda_{n}(\underline{\omega})$ are simple, and condition (A3) is equivalent to requiring the diophantine condition

$$
|\langle\underline{\omega}, \underline{v}\rangle|>\Lambda^{-1}|\underline{v}|^{-\gamma}, \quad \gamma>s-1, \underline{m}-\underline{n}:=\underline{v} \neq 0
$$

on the frequencies $\underline{\omega}$. The set of the diophantine values of $\underline{\omega}$ is dense in $[0,1]^{s}$.

By condition (1-20), the eigenvalues $\lambda_{\underline{n}}(\underline{\omega})$ are simple. The corresponding normalized eigenvectors are

$$
\psi_{\underline{n}}(\underline{x})=\frac{1}{\sqrt{2^{\underline{n}} \underline{n} !}} e^{-\underline{x}^{2} / 2} H_{\underline{n}}(\underline{x}):=|\underline{n}\rangle,
$$

where $x \mapsto H_{n}(x), n=0,1, \ldots$, is the $n$-th Hermite polynomial in $\mathbb{R}$ and

$$
\begin{aligned}
\underline{x} & =\left(x_{1}, \ldots, x_{s}\right) \in \mathbb{R}^{s}, & 2^{\underline{n}} & =2^{n_{1}+\cdots+n_{s}}, \\
e^{-\underline{x}^{2}} & =e^{-x_{1}^{2}-\cdots-x_{s}^{2}}, & H_{\underline{n}}(\underline{x}) & =H_{n_{1}}\left(x_{1}\right) \cdots H_{n_{s}}\left(x_{s}\right) .
\end{aligned}
$$


Now let $2<q<6$. Consider the function $\mathcal{V}(\underline{x}) \in C^{\infty}\left(\mathbb{R}^{s} ; \mathbb{R}\right)$ such that

$$
\mathcal{V}(\underline{x})=e^{-|\underline{x}|^{q}} \Phi(\underline{x}), \quad \sup _{\underline{x} \in \mathbb{R}^{s}}|\Phi(\underline{x})| \leq 1,|\underline{x}|^{q}:=\left|x_{1}\right|^{q}+\cdots+\left|x_{s}\right|^{q} .
$$

Denote by $V(\underline{x})$ the maximal multiplication operator by $\mathcal{V}(\underline{x})$ in $L^{2}\left(\mathbb{R}^{s}\right)$. Then $\|V\|_{L^{2} \rightarrow L^{2}} \leq 1$. Thus, the operator $H=T+V$ defined on $D(T)$ is self-adjoint in $L^{2}\left(\mathbb{R}^{s}\right)$ with compact resolvent. In Proposition 2.6 below we will prove the estimate

$$
\left|\left\langle\psi_{\underline{m}}, V \psi_{\underline{n}}\right\rangle_{L^{2}\left(\mathbb{R}^{s}\right)}\right| \leq e^{-\Sigma(|\underline{m}|+|\underline{n}|)},
$$

with $\Sigma>0$ independent of $(\underline{m}, \underline{n})$. Hence, (A4) is fulfilled in this example.

\section{Proof of the result}

We recall the basic definitions of (Rayleigh-Schrödinger) eigenvalue perturbation theory [Kato 1976, §II.1.5 and §§VII.1-3] in this context. Consider an eigenvalue $\lambda_{\underline{n}}, \underline{n} \in \mathbb{Z}^{s}$, of $T$ corresponding to the (normalized) eigenvector $\psi_{\underline{n}}$, henceforth abbreviated $|\underline{n}\rangle$.

- $P_{\underline{n}}$ denotes the (one-dimensional) orthogonal projection operator from $X$ to the one-dimensional subspace spanned by $|\underline{n}\rangle$.

- $S$ denotes the reduced resolvent of $T$, i.e., $S(\lambda):=\sum_{\underline{k} \neq \underline{n} \in \mathbb{Z}^{s}} \frac{P_{\underline{k}}}{\lambda_{\underline{k}}-\lambda}$.

- $S_{\underline{n}}$ is the reduced resolvent evaluated at $\lambda=\lambda_{\underline{n}}$, i.e., $S_{\underline{n}}:=\sum_{\underline{k} \neq \underline{n} \in \mathbb{Z}^{s}} \frac{P_{\underline{k}}}{\lambda_{\underline{k}}-\lambda_{\underline{n}}}$.

$$
\left[S_{\underline{n}}\right]^{\ell}:=\sum_{\underline{k} \neq \underline{n} \in \mathbb{Z}^{s}} \frac{P_{\underline{k}}}{\left(\lambda_{\underline{k}}-\lambda_{\underline{n}}\right)^{\ell}}, \quad \ell \geq 1, \quad\left[S_{\underline{n}}\right]^{0}=P_{\underline{n}} .
$$

In this situation we can use the explicit expressions (2.32)-(2.33) in [Kato 1976, Chapter II] for the coefficients $B_{\ell}(\underline{n})$ of the perturbation series (1-7):

$$
B_{\ell}(\underline{n})=\sum_{p=1}^{\ell} \frac{(-1)^{p}}{p} \sum_{\substack{k_{1}+\ldots+k_{p}=p-1 \\ k_{i}=0,1, \ldots \\ i=1, \ldots, p-1}} \operatorname{Tr}\left[V\left[S_{\underline{n}}\right]^{k_{1}} V \cdots V\left[S_{\underline{n}}\right]^{k_{p}}\right] .
$$

Since

$$
\begin{gathered}
{\left[S_{\underline{n}}\right]^{k_{p}}|\underline{n}\rangle=0, \quad k_{p}>0, \quad\left[S_{\underline{n}}\right]^{0}|\underline{n}\rangle=|\underline{n}\rangle,} \\
\operatorname{Tr}\left[V\left[S_{\underline{n}}\right]^{k_{1}} V \cdots V\left[S_{\underline{n}}\right]^{k_{p}}\right]=\left\langle n, V\left[S_{\underline{n}}\right]^{k_{1}} V \cdots V^{k_{p}} n\right\rangle,
\end{gathered}
$$


(2-2) becomes

$$
B_{\ell}(\underline{n})=\sum_{p=1}^{\ell} \frac{(-1)^{p}}{p} B_{\ell, p}(\underline{n}),
$$

where

$$
B_{\ell, p}(\underline{n})=\sum_{k_{1}+\cdots+k_{p-1}=p-1}\left\langle\underline{n}, V\left[S_{\underline{n}}\right]^{k_{1}} V \cdots V\left[S_{\underline{n}}\right]^{k_{p-1}} V \underline{n}\right\rangle .
$$

Remark 2.1. For $\ell=2$, we have $p=2$ and the above formulas yield the standard second-order term of the Rayleigh-Schrdinger expansion:

$$
B_{2,2}(\underline{n})=\sum_{\underline{k} \neq \underline{n}} \frac{|\langle\underline{n}, V \underline{k}\rangle|^{2}}{\lambda_{\underline{k}}-\lambda_{\underline{n}}} .
$$

In the absence of a condition controlling the vanishing of $\left|\lambda_{\underline{k}}-\lambda_{\underline{n}}\right|$ as $|\underline{k}-\underline{n}| \rightarrow \infty$, the above series can of course diverge even if

$$
\sup _{\underline{n} \in \mathbb{N}^{s}} \sum_{\underline{k} \in \mathbb{N}^{s}}\langle|\underline{n}, V \underline{k}|\rangle^{2}<+\infty
$$

On the other hand, this last inequality is implied by the standard Schur condition ensuring the boundedness of $V^{2}$ and hence of $V$.

We can rewrite in more detail the factor $\left\langle\underline{n}, V S_{\underline{n}}^{k_{1}} \cdots V S_{\underline{n}}^{k_{p-1}} V \underline{n}\right\rangle$ making explicit all factors with at least one $k_{i}=0$. Namely, consider the $q$-partition

$$
p-1=j_{1}+\cdots+j_{q}, \quad j_{s} \geq 1,1 \leq q \leq p-1 .
$$

The number of the $q$-partitions is (see, e.g., [Andrews 1976]) $N(p, q)=\left(\begin{array}{c}p-1 \\ q-1\end{array}\right)$. Furthermore, let $m(p, q)=p-1-q$ be the total number of zeros in the $q$-partition of $\left(k_{1}, \ldots, k_{p-1}\right)$. We can thus rearrange the sequence $\left(k_{1}, \ldots, k_{p-1}\right)$ as

$$
k_{1}+\cdots+k_{p-1}=p-1=m_{1}+j_{1}+m_{2}+j_{2}+m_{q}+j_{q}, \quad m=m_{1}+\cdots+m_{q} .
$$

As a consequence,

$$
\begin{aligned}
V\left[S_{\underline{n}}\right]^{k_{1}} \cdots V\left[S_{\underline{n}}\right]^{k_{p-1}} V= & \sum_{q=1}^{(p-1)} N(p, q) \prod_{h=1}^{m_{1}}\left[V P_{\underline{n}}\right]^{h} V\left[S_{\underline{n}}\right]^{j_{1}} \\
& \times \prod_{h=1}^{m_{2}}\left[V P_{\underline{n}}\right]^{h} \cdot V\left[S_{\underline{n}}\right]^{j_{2}} \cdots \prod_{h=1}^{m_{q}}\left[V P_{\underline{n}}\right]^{h} \cdot V\left[S_{\underline{n}}\right]^{j_{q}} V,
\end{aligned}
$$

whence: 
Lemma 2.2. Let $k_{1}, \ldots, k_{p}, m_{1}, \ldots, m_{q}, j_{1}, \ldots, j_{q}$ be related through (2-7). Then

$$
\begin{aligned}
& B_{\ell, p}(\underline{n})=\sum_{k_{1}+\cdots+k_{p-1}=p-1}\left\langle\underline{n}, V\left[S_{\underline{n}}\right]^{k_{1}} \cdots V\left[S_{\underline{n}}\right]^{k_{p-1}} V \underline{n}\right\rangle \\
& =\sum_{q=1}^{p-1} N(p, q)\langle\underline{n}, V \underline{n}\rangle^{m_{1}+\cdots+m_{q}} \sum_{\substack{\underline{r}_{1} \neq n, \ldots, \underline{r}_{q} \neq \underline{n} \\
j_{1}+\cdots+j_{q}=p-1}} \frac{\left|\left\langle\underline{n}, V \underline{r}_{1}\right\rangle\right|^{2}}{\left(\lambda_{\underline{r}_{1}}-\lambda_{\underline{n}}\right)^{j_{1}}} \cdot \frac{\left|\left\langle\underline{n}, V \underline{r}_{2}\right\rangle\right|^{2}}{\left(\lambda_{\underline{r}_{2}}-\lambda_{\underline{n}}\right)^{j_{2}}} \cdots \frac{\left|\left\langle\underline{n}_{\underline{n}}, V \underline{r}_{q}\right\rangle\right|^{2}}{\left(\lambda_{\underline{r}_{q}}-\lambda_{\underline{n}}\right)^{j_{q}}} \\
& =\sum_{q=1}^{p-1} N(p, q)\langle\underline{n}, V \underline{n}\rangle^{p-1-q} \sum_{\substack{\underline{r}_{1} \neq \underline{n}, \ldots, \underline{r}_{q} \neq \underline{n} \\
j_{1}+\cdots+j_{q}=p-1}} \frac{\left|\left\langle\underline{n}, V \underline{r}_{1}\right\rangle\right|^{2}}{\left(\lambda_{\underline{r}_{1}}-\lambda_{\underline{n}}\right)^{j_{1}}} \cdot \frac{|\langle\underline{n}, V \underline{\underline{r}} 2\rangle|^{2}}{\left(\lambda_{\underline{r}_{2}}-\lambda_{\underline{n}}\right)^{j_{2}}} \cdots \frac{\left|\left\langle\underline{n}, V \underline{r}_{q}\right\rangle\right|^{2}}{\left(\lambda_{\underline{r}_{q}}-\lambda_{\underline{\underline{n}}}\right)^{j_{q}}} .
\end{aligned}
$$

Proof. The product (2-8) is unchanged if $P_{\underline{n}}$ is replaced by $P_{n}^{2}$. Recalling that

$$
S^{j}=\sum_{\underline{r} \neq \underline{n}}^{\infty} \frac{P_{\underline{r}}}{\left(\lambda_{\underline{r}}-\lambda_{\underline{n}}\right)^{j}}
$$

and denoting

$$
\begin{aligned}
\Omega_{1}(m, j ; n) & :=\prod_{h=1}^{m}\left[V P_{\underline{n}}^{2}\right]^{h} V S^{j}, \\
\Omega_{2}(m-1, j ; n) & :=\prod_{h=1}^{m-1}\left[V P_{\underline{n}}^{2}\right]^{h} V P_{\underline{n}} P_{\underline{n}} V S^{j}, \\
\Omega_{3}(m-1, j ; n, r) & :=\prod_{h=1}^{m-1}\left[V P_{\underline{n}}^{2}\right]^{h} V P_{\underline{n}} P_{\underline{n}} V P_{\underline{r}} P_{\underline{r}},
\end{aligned}
$$

this yields

$$
\begin{aligned}
& B_{\ell, p}(\underline{n})=\sum_{q=1}^{p-1} N(p, q)\left\langle\underline{n}, \Omega_{1}\left(m_{1}, j_{1} ; n\right) \cdots \Omega_{1}\left(m_{q}, j_{q} ; n\right) V \underline{n}\right\rangle \\
& =\sum_{q=1}^{p-1} N(p, q)\left\langle\underline{n}, \prod_{h=1}^{m_{1}-1} \Omega_{2}\left(m_{1}-1, j_{1} ; n\right) \cdots \Omega_{2}\left(m_{q}-1, j_{q} ; n\right) V \underline{n}\right\rangle \\
& =\sum_{q=1}^{p-1} N(p, q)\left(\lambda_{\underline{r}_{1}}-\lambda_{\underline{n}}\right)^{-j_{1}} \cdot\left(\lambda_{\underline{r}_{2}}-\lambda_{\underline{n}}\right)^{-j_{2}} \cdots\left(\lambda_{\underline{r}_{q}}-\lambda_{\underline{n}}\right)^{-} j_{q} \\
& \times \sum_{\substack{\underline{r}_{1} \neq \underline{n}, \ldots, \underline{r}_{q} \neq \underline{n} \\
j_{1}+\cdots+j_{q}=p-1}}\left\langle\underline{n}, \Omega_{3}\left(m_{1}-1, j_{1} ; n, \underline{r}_{1}\right) \cdots \Omega_{3}\left(m_{q}-1, j_{q} ; n, \underline{r}_{q}\right) V \underline{n}\right\rangle .
\end{aligned}
$$


Hence, by (2-8),

$$
\begin{aligned}
& \sum_{k_{1}+\cdots+k_{p-1}=p-1}\left\langle n, V\left[S_{\underline{n}}\right]^{k_{1}} \cdots V\left[S_{\underline{n}}\right]^{k_{p-1}} V n\right\rangle \\
& =\sum_{q=1}^{p-1} N(p, q)\left\langle\underline{n}, \prod_{h=1}^{m_{1}-1}\left[V P_{\underline{n}}^{2}\right]^{h} V P_{\underline{n}} P_{\underline{n}} V P_{\underline{r}_{1}} P_{\underline{r}_{1}}\right. \\
& \left.\times \prod_{h=1}^{m_{2}-1}\left[V P_{\underline{n}}^{2}\right]^{h} V P_{\underline{n}} P_{\underline{n}} \cdot V P_{\underline{r}_{2}} P_{\underline{r}_{2}} \cdots \prod_{h=1}^{m_{q}} V P_{\underline{r}_{q}} P \underline{r}_{q} V \underline{n}\right) \\
& =\sum_{q=1}^{p-1} N(p, q)\langle\underline{n}, V \underline{n}\rangle^{m_{1}} \cdots\langle\underline{n}, V \underline{n}\rangle^{m_{q}} \\
& \times \sum_{\underline{r}_{1} \neq \underline{n}, \ldots, \underline{r}_{q} \neq \underline{n}} \frac{\left\langle\underline{n}, V \underline{r}_{1}\right\rangle\left\langle\underline{r}_{1}, V \underline{n}\right\rangle}{\left(\lambda_{\underline{r}_{1}}-\lambda_{\underline{n}}\right)^{j_{1}}} \cdot \frac{\left\langle\underline{n}, V \underline{r}_{2}\right\rangle\left\langle\underline{r}_{2}, V \underline{n}\right\rangle}{\left(\lambda_{\underline{r}_{2}}-\lambda_{\underline{n}}\right)^{j_{2}}} \cdots \frac{\left\langle\underline{n}, V \underline{r}_{q}\right\rangle\left\langle\underline{r}_{q}, V \underline{n}\right\rangle}{\left(\lambda_{\underline{r}_{q}}-\lambda_{\underline{n}}\right)^{j_{q}}} \\
& =\sum_{q=1}^{p-1} N(p, q)\langle\underline{n}, V \underline{n}\rangle^{p-1-q} \sum_{\substack{r_{1} \neq \underline{n}, \ldots, r_{q} \neq \underline{n} \\
j_{1}+\cdots+j_{q}=p-1}} \frac{\left|\left\langle\underline{n}, V \underline{r}_{1}\right\rangle\right|^{2}}{\left(\lambda_{\underline{r_{1}}}-\lambda_{\underline{n}}\right)^{j_{1}}} \cdot \frac{\left|\left\langle\underline{n}, V \underline{r}_{2}\right\rangle\right|^{2}}{\left(\lambda_{\underline{r}_{2}}-\lambda_{\underline{n}}\right)^{j_{2}}} \cdots \frac{\left|\left\langle\underline{n}, V \underline{\underline{r}_{q}}\right\rangle\right|^{2}}{\left(\lambda_{\underline{r}_{q}}-\lambda_{\underline{n}}\right)^{j_{q}}} .
\end{aligned}
$$

This concludes the proof.

The first step in estimating the coefficients $B_{\ell}(n)$ is therefore estimating the fractions $|\langle\underline{n}, V \underline{r}\rangle|^{2} /\left(\lambda_{\underline{r}}-\lambda_{\underline{n}}\right)^{j}$. In turn, this requires an analysis of the vanishing mechanism of the denominators $\left(\lambda_{\underline{r}}-\lambda_{\underline{r}}\right)^{j}$. A preliminary remark is:

Lemma 2.3. With the assumptions of Lemma 2.2,

$$
\sum_{\underline{r} \neq \underline{n} \in \mathbb{Z}^{s}} \frac{\left|\langle\underline{n}, V \underline{r}\rangle^{2}\right|}{\left|\lambda_{\underline{r}}-\lambda_{\underline{n}}\right|^{j}} \leq \frac{p A}{\alpha^{p}}\left(\frac{p \Lambda}{\alpha^{\gamma}}\right)^{j}(\gamma j) !
$$

Proof. Equation (2-9) is a direct consequence of assumptions (A3) and (A4) because

$$
\sum_{\underline{r} \neq \underline{n} \in \mathbb{Z}^{s}} \frac{\left|\langle\underline{n}, V \underline{r}\rangle^{2}\right|}{\left|\lambda_{\underline{r}}-\lambda_{\underline{n}}\right|^{j}} \leq A \Lambda^{j} \sum_{\underline{r} \neq \underline{n} \in \mathbb{Z}^{s}}|\underline{r}-\underline{n}|^{\gamma j} e^{-\alpha|\underline{r}-\underline{n}|}=A \Lambda^{j} \sum_{\underline{x} \neq 0}|\underline{x}|^{\gamma j} e^{-\alpha|\underline{x}|},
$$

where

$\sum_{\underline{x} \neq 0}|\underline{x}|^{\gamma j} e^{-\alpha|\underline{x}|}:=\sum_{\underline{x} \neq 0}\left[\left|x_{1}\right|^{\gamma}+\cdots+\left|x_{p}\right|^{\gamma}\right]^{j} e^{-\alpha|\underline{x}|} \leq p^{j}\left(\left|x_{1}\right|^{\gamma j}+\cdots+\left|x_{p}\right|^{\gamma j}\right) e^{-\alpha|\underline{x}|}$.

Hence,

$$
\sum_{\underline{x} \neq 0}|\underline{x}|^{\gamma j} e^{-\alpha|\underline{x}|} \leq p^{j+1} \sum_{x_{1} \neq 0}\left|x_{1}\right|^{\gamma j} e^{-\alpha|\underline{x}|} \leq \frac{p^{j+1}(\gamma j) !}{\alpha^{\gamma j+p}}
$$


and summing up we get

$$
\sum_{\underline{r} \neq \underline{n} \in \mathbb{Z}^{s}} \frac{\left|\langle\underline{n}, V \underline{r}\rangle^{2}\right|}{\left|\lambda_{\underline{r}}-\lambda_{\underline{n}}\right|^{j}} \leq \frac{p A}{\alpha^{p}}\left(\frac{p \Lambda}{\alpha^{\gamma}}\right)^{j}(\gamma j) !
$$

and this proves the lemma.

Corollary 2.4. Recalling that $k_{1}+\cdots+k_{p-1}=p-1, p=2, \ldots$, in the assumptions of Lemma 2.3, the following bounds hold:

$$
\begin{aligned}
\left|B_{\ell, p}(\underline{n})\right| & \leq \sum_{k_{1}+\cdots+k_{p-1}=p-1}\left|\left\langle n, V\left[S_{\underline{n}}\right]^{k_{1}} \cdots V\left[S_{\underline{n}}\right]^{k_{p-1}} V n\right\rangle\right| \\
& \leq p\left(\frac{2 A}{\alpha}\right)^{p}\left(\frac{p \Lambda}{\alpha^{\gamma}}\right)^{p-1} \sum_{q=1}^{p-1} p^{q} \sum_{j_{1}+\cdots+j_{q}=p-1}\left(\gamma j_{1}\right) ! \cdots\left(\gamma j_{q}\right) ! .
\end{aligned}
$$

Proof. It is enough to insert (2-9) in the statement of Lemma 2.2 on account of the bounds $N(p, q)<2^{p},|\langle\underline{n}, V \underline{n}\rangle| \leq 1$ and the fact that $j_{1}+\cdots+j_{q}=p-1$.

We can now state and prove the main estimate.

Proposition 2.5. Under assumptions (A1)-(A3),

$$
\left|B_{\ell}(\underline{n})\right| \leq\left(4 \ell^{2}\right)^{\ell+1} R(\Lambda, \alpha, \gamma)^{\ell}[\gamma(\ell-1)] ! .
$$

Proof. We have, by (2-4),

$$
\left|B_{\ell}(\underline{n})\right| \leq \sum_{p=1}^{\ell} \frac{B_{\ell, p}(\underline{n})}{p}
$$

Clearly,

Moreover,

$$
\sum_{j_{1}+\cdots+j_{q}=p-1}\left(\gamma j_{1}\right) ! \cdots\left(\gamma j_{q}\right) ! \leq(p-1)[\gamma(p-1)] ! .
$$

$$
\sum_{q=1}^{p-1} p^{q} \leq p^{p}
$$

Therefore, by Corollary 2.4,

$$
\left|B_{\ell, p}(\underline{n})\right|<(2 p)^{p}(p-1) p\left(\frac{2 A}{\alpha}\right)^{p}\left(\frac{p \Lambda}{\alpha^{\gamma}}\right)^{p-1}[\gamma(p-1)] !,
$$

whence since $A<\alpha$

$$
\begin{aligned}
\left|B_{\ell}(\underline{n})\right| & \leq \sum_{p=1}^{\ell} B_{\ell, p}(\underline{n}) \leq(2 \ell)^{\ell} \ell^{3} 2^{\ell} \ell^{\ell-1}\left(\frac{\Lambda}{\alpha^{\gamma}}\right)^{\ell-1}[\gamma(\ell-1)] ! \\
& \leq\left(4 \ell^{2}\right)^{\ell+1} R(\Lambda, \alpha, \gamma)^{\ell}[\gamma(\ell-1)] !,
\end{aligned}
$$


where

$$
R(\Lambda, \alpha, \gamma):=\frac{\Lambda}{\alpha^{\gamma}}
$$

Thus, the proof of the proposition is complete.

Proof of Theorem 1.1. The assertion is just (2-11).

Proof of Corollary 1.2. The validity of (1-11) is a direct consequence of the existence of the perturbation expansion for all $\underline{n} \in \mathbb{N}^{p}$. The uniformity with respect to $\underline{n}$ follows from the $\underline{n}$-independent bound (1-10).

Example 1.5 (continued). Consider again the normalized eigenvectors of $T$ :

$$
\psi_{\underline{n}}(\underline{x})=\prod_{k=1}^{s} \psi_{n_{k}}\left(x_{k}\right), \quad \psi_{n}(x):=\frac{1}{\sqrt{2^{n} n !}} e^{-x^{2} / 2} H_{n}(x), n=0,1, \ldots,
$$

where $H_{n}(x), x \in \mathbb{R}$, is the $n$-th Hermite polynomial. The vectors $\left\{\psi_{n}(\underline{x}): \underline{n} \in \mathbb{N}^{s}\right\}$ form an orthonormal basis in $L^{2}\left(\mathbb{R}^{s}\right)$. Recall that

$$
\mathcal{V}(\underline{x}):=e^{-|\underline{x}|^{q}} \Phi(\underline{x}) .
$$

Consequently, considering the potential $\mathcal{V}(\underline{x})$ and the corresponding maximal multiplication operator $V$ in $L^{2}\left(\mathbb{R}^{s}\right)$, we have:

Proposition 2.6. Condition (A4) is fulfilled in this example; i.e., there is $\Sigma(q)>0$ such that

$$
\left\langle\psi_{\underline{m}}, V \psi_{\underline{n}}\right\rangle:=\langle\underline{m}, \mathcal{V}(\underline{x}) \underline{n}\rangle \leq e^{-\Sigma[|\underline{m}|+|\underline{n}|]} .
$$

Proof. Consider first the case $s=1$. Recall the formula

$$
H_{n}(x)=n ! \sum_{k=0}^{[n / 2]} \frac{(-1)^{k}(2 x)^{n-2 k}}{k !(n-2 k) !}
$$

where as usual $[n / 2]$ is the integer part of $n$. We can thus write

$$
\psi_{m}(x) \psi_{n}(x)=\sqrt{2^{-(m+n) / 2} m ! n !} \sum_{h=0}^{[m / 2]} \sum_{k=0}^{[n / 2]} \frac{(-1)^{h+k}(2 x)^{m-2 h}(2 x)^{n-2 k}}{h !(m-2 h) ! k !(n-2 k) !},
$$

whence

$$
\begin{aligned}
|\langle m, \mathcal{V} n\rangle| & \leq \sqrt{2^{(m+n) / 2} m ! n !} \sum_{h=0}^{[m / 2]} \sum_{k=0}^{[n / 2]} \frac{\int_{R} x^{m+n-2(h+k)} e^{-|x|^{q}} d x}{h !(m-2 h) ! k !(n-2 k) !} \\
& <\sqrt{2^{(m+n) / 2} m ! n !} \sum_{h=0}^{[m / 2]} \sum_{k=0}^{[n / 2]} \frac{\Gamma([m+n-2(h+k)+1] / q)}{h !(m-2 h) ! k !(n-2 k) !} .
\end{aligned}
$$


Now,

$$
\begin{aligned}
\min _{0 \leq h \leq[m / 2]}[h !(m-2 h) !] & =\Gamma(m / 3+1)^{2}, \\
\min _{0 \leq k \leq[n / 2]}[h !(n-2 k) !] & =\Gamma(n / 3+1)^{2}, \\
\max _{\substack{0 \leq h \leq[m / 2] \\
0 \leq k \leq[n / 2]}}[\Gamma([m+n-2(h+k)+1] / q)] & =\Gamma([m+n+1] / q),
\end{aligned}
$$

and this implies

$$
|\langle m, \mathcal{V} n\rangle| \leq \sqrt{2^{(m+n) / 2} m ! n !} \cdot\left[\frac{m}{2}\right] \cdot\left[\frac{n}{2}\right] \frac{\Gamma([m+n+1] / q)}{\Gamma(m / 3+1)^{2} \cdot \Gamma(n / 3+1)^{2}} .
$$

Now apply the Stirling formula. Since

$$
6 \pi 2^{(m+n) / 4} e^{-(m+n) / 2} e^{-[m+n+1] / q} e^{(m+n) / 3} \cdot\left[\frac{m}{2}\right] \cdot\left[\frac{n}{2}\right] \leq 1
$$

for $m+n$ large enough,

$$
|\langle m, \mathcal{V} n\rangle| \leq \frac{((m+q) / q)^{(m+q) / q}}{m^{m / 6} n^{n / 6}} .
$$

Without loss, we can take $m=n+k, k \geq 0$. Then

$$
|\langle m, \mathcal{V} n\rangle|=|\langle n, \mathcal{V}(n+k)\rangle| \leq \frac{[(2 n+k) / q]^{(2 n+k) / q}}{(n+k)^{(n+k) / 6} n^{n / 6}}
$$

Now $(n+k)>(2 n+k) / q>(n+k) / 6$ if $2<q<6$ and hence there is $0<L<1$ such that

$$
|\langle n, \mathcal{V}(n+k)\rangle| \leq \frac{[(2 n+k) / q]^{(n+k) / 6}}{(n+k)^{(n+k) / 6} n^{n / 6}}<\frac{L^{n+k}}{n^{n / 6}}=\frac{L^{m}}{n^{n / 6}},
$$

whence, a fortiori, with $L=e^{-\Sigma}$

$$
|\langle m, \mathcal{V} n\rangle| \leq e^{-\Sigma(m+n)} .
$$

This concludes the proof for $s=1$. The general case follows through an immediate product argument.

\section{References}

[Andrews 1976] G. E. Andrews, The theory of partitions, Cambridge University, 1976.

[Brownell and Clark 1961] F. H. Brownell and C. W. Clark, "Asymptotic distribution of the eigenvalues of the lower part of the Schrödinger operator spectrum”, J. Math. Mech. 10:1 (1961), 31-70.

[Kato 1976] T. Kato, Perturbation theory for linear operators, 2nd ed., Grundlehren der mathematischen Wissenschaften 132, Springer, Berlin, 1976.

[McLeod 1961] J. B. McLeod, "The distribution of the eigenvalues for the hydrogen atom and similar cases", Proc. London Math. Soc. (3) 11 (1961), 139-158. 
[Reed and Simon 1978] M. Reed and B. Simon, Methods of modern mathematical physics, IV: Analysis of operators, Academic, San Diego, 1978.

[Tamura 1974] H. Tamura, "The asymptotic distribution of the lower part eigenvalues for elliptic operators”, Proc. Japan Acad. 50 (1974), 185-187.

Received 26 Oct 2015. Revised 28 Oct 2015. Accepted 9 May 2016.

FIORELLA BARONE: fiorella.barone@uniba.it

Dipartimento di Matematica, Università di Bari, 70122 Bari, Italy

SANDRO GRAFFI: sandro.graffi@unibo.it

Dipartimento di Matematica, Università di Bologna, 40127 Bologna, Italy

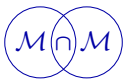


EDITORIAL BOARD

ANTONIO CARCATERRA

ERIC A. CARLEN

FRANCESCO DELL'ISOLA

RAFFAELE ESPOSITO

ALBERT FANNJIANG

Gilles A. FranCFORT

Pierangelo MARCATI

JEAN-JACQUES MARIGO

PETER A. MARKOWICH

MARTIN OSTOJA-STARZEWSKI

PIERRE SEPPECHER

DAVID J. STEIGMANN

PAUl STEINMANN

PierRe M. SuQueT

MANAGING EDITORS

MICOL AMAR

CORRADO LATTANZIO

ANGELA MADEO

MARTIN OSTOJA-STARZEWSKI

ADVISORY BOARD

ADNAN AKAY

Holm AltenBaCH

MICOL AMAR

HARM ASKES

TEODOR ATANACKOVIĆ

VICTOR BERDICHEVSKY

GUY BOUCHITTÉ

ANDREA BRAIDES

ROBERTO CAMASSA

MAURO CARFORE

ERIC DARVE

FELIX DARVE

ANNA DE MASI

GianPiEtro DEL Piero

EMMANUELE Di BENEDETTO

BERNOLD FIEDLER

IRENE M. GAMBA

DAVID Y. GAO

SERGEY GAVRILYUK

TIMOTHY J. HEALEY

DOMINIQUE JEULIN

ROGER E. KHAYAT

CORRADO LATTANZIO

ROBERT P. LIPTON

ANGELO LUONGO

ANGELA MADEO

JUAN J. MANFREDI

CARLO MARCHIORO

GÉRARD A. MAUGIN

ROBERTO NATALINI PATRIZIO NEFF

ANDREY PIATNITSKI

ERRICO PRESUTTI

MARIO PULVIRENTI

LUCIO RUSSO

Miguel A. F. SANJUAN

PATRICK SElVADURAI

ALEXANDER P. SEYRANIAN

MIROSLAV ŠILHAVÝ

GUIDO SWEERS

ANTOINETTE TORDESILLAS

LEV TRUSKINOVSKY

JUAN J. L. VELÁZQUEZ VINCENZO VESPRI ANGELO VULPIANI msp.org/memocs

Università di Roma "La Sapienza", Italia

Rutgers University, USA

(CO-CHAIR) Università di Roma "La Sapienza", Italia

(TREASURER) Università dell'Aquila, Italia

University of California at Davis, USA

(CO-CHAIR) Université Paris-Nord, France

Università dell'Aquila, Italy

École Polytechnique, France

DAMTP Cambridge, UK, and University of Vienna, Austria

(CHAIR MANAGING EDITOR) Univ. of Illinois at Urbana-Champaign, USA

Université du Sud Toulon-Var, France

University of California at Berkeley, USA

Universität Erlangen-Nürnberg, Germany

LMA CNRS Marseille, France

Università di Roma "La Sapienza", Italia

Università dell'Aquila, Italy

Université de Lyon-INSA (Institut National des Sciences Appliquées), France

(CHAIR MANAGING EDITOR) Univ. of Illinois at Urbana-Champaign, USA

Carnegie Mellon University, USA, and Bilkent University, Turkey

Otto-von-Guericke-Universität Magdeburg, Germany

Università di Roma "La Sapienza", Italia

University of Sheffield, UK

University of Novi Sad, Serbia

Wayne State University, USA

Université du Sud Toulon-Var, France

Università di Roma Tor Vergata, Italia

University of North Carolina at Chapel Hill, USA

Università di Pavia, Italia

Stanford University, USA

Institut Polytechnique de Grenoble, France

Università dell'Aquila, Italia

Università di Ferrara and International Research Center MEMOCS, Italia

Vanderbilt University, USA

Freie Universität Berlin, Germany

University of Texas at Austin, USA

Federation University and Australian National University, Australia

Université Aix-Marseille, France

Cornell University, USA

École des Mines, France

University of Western Ontario, Canada

Università dell' Aquila, Italy

Louisiana State University, USA

Università dell'Aquila, Italia

Université de Lyon-INSA (Institut National des Sciences Appliquées), France University of Pittsburgh, USA

Università di Roma "La Sapienza”, Italia

Université Paris VI, France

Istituto per le Applicazioni del Calcolo "M. Picone", Italy

Universität Duisburg-Essen, Germany

Narvik University College, Norway, Russia

Università di Roma Tor Vergata, Italy

Università di Roma "La Sapienza”, Italia

Università di Roma “Tor Vergata”, Italia

Universidad Rey Juan Carlos, Madrid, Spain

McGill University, Canada

Moscow State Lomonosov University, Russia

Academy of Sciences of the Czech Republic

Universität zu Köln, Germany

University of Melbourne, Australia

École Polytechnique, France

Bonn University, Germany

Università di Firenze, Italia

Università di Roma La Sapienza, Italia

MEMOCS (ISSN 2325-3444 electronic, 2326-7186 printed) is a journal of the International Research Center for the Mathematics and Mechanics of Complex Systems at the Università dell'Aquila, Italy.

Cover image: "Tangle” by $\odot$ John Horigan; produced using the Context Free program (contextfreeart.org).

PUBLISHED BY

7 mathematical sciences publishers

nonprofit scientific publishing

http://msp.org/

(C) 2016 Mathematical Sciences Publishers 
Special issue in honor of

Lucio Russo

Lucio Russo: A multifaceted life

Raffaele Esposito and Francesco dell'Isola

The work of Lucio Russo on percolation Geoffrey R. Grimmett

"Mathematics" and "physics" in the science of harmonics Stefano Isola

From quantum to classical world: emergence of trajectories in a quantum system Rodolfo Figari and Alessandro Teta

Propagation of chaos and effective equations in kinetic theory: a brief survey Mario Pulvirenti and Sergio Simonella

What decides the direction of a current? Christian Maes

A remark on eigenvalue perturbation theory at vanishing isolation distance Fiorella Barone and Sandro Graffi

Some results on the asymptotic behavior of finite connection probabilities in percolation Massimo Campanino and Michele Gianfelice

Correlation inequalities for the Potts model Geoffrey R. Grimmett

Quantum mechanics: some basic techniques for some basic models, I: The models Vincenzo Grecchi

Quantum mechanics: some basic techniques for some basic models, II: The techniques Vincenzo Grecchi

On stochastic distributions and currents

Vincenzo Capasso and Franco Flandoli

A note on Gibbs and Markov random fields with constraints and their moments Alberto Gandolfi and Pietro Lenarda

Quantum mechanics: light and shadows (ontological problems and epistemic solutions) Gianfausto Dell'Antonio

MEMOCS is a journal of the International Research Center for the Mathematics and Mechanics of Complex Systems at the Università dell' Aquila, Italy.

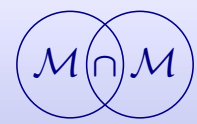

